\title{
Effect of the Modification of the Free Carboxylic Acid Groups of an HPLC Chiral Stationary Phase Based on (+)-(18-Crown-6)-2,3,11,12-tetracarboxylic Acid on the Chiral Recognition
}

\author{
Hwan Sun Cho, Hee Jung Choi, Areum Lee, Jong Sung Jin, ${ }^{*}$ Euh Duck Jeong," and Myung Ho Hỵu" \\ Department of Chemistry and Chemistry Institute for Functional Materials, Pusan Vational Lniversity. \\ Pusan 609-735, Korea. "E-mail: mhinuntapusan.ac.kr \\ ${ }^{\dagger}$ High Technologv Components \& Materials Research Team. Korea Basic Science Institute, Busan 618-230, Korea \\ Received Mav 29. 2009. Accepted June 29, 2009
}

Key Words: Chiral stationary phase. Liquid chromatography, Chiral recognition, (+)-(18-Crown-6)-2,3. 11,12-tetracarboxylic acid

Chiral stationary phases (CSPs) based on chiral crown ethers have been known to be quite effective in the liquid chromatographic resolution of racemic compounds containing a primary amino group. ${ }^{3}$ For example, (+)-(18-crown-6)-2,3, 11.12-tetracarboxylic acid covalently bonded to silica gel has been successfully utilized as CSPs for the liquid chromatographic resolution of racemic compounds containing a primary amino group. "Especially CSP 1 (Figure 1) developed in our laboratory by bonding $(+)-(18-c r o w n-6)-2,3,11,12$ tetracarboxylic acid to 3-aminopropylsilica gel was very successful in resolving various racemic primary amino compounds including $\alpha-\beta$ - and $\gamma$-amino acids, ${ }^{3}$ aryl $\alpha$-aminoketones. ${ }^{+}$tocainide and its analogues. ${ }^{5}$ racemic amines. racemic amino alcolols. ${ }^{6} d i$ and $t r i$-peptides and racemic fluoroquinolone antibacterials. ${ }^{2 \mathrm{~b} .8}$

Even though the chiral recognition mechanism for the resolution of racemic primary amino compounds by CSP 1 is not clear yet. the enantioselective complexation of the primary ammonium ions ( $\mathrm{R}^{-\mathrm{NH}_{3}}{ }^{-}$) of analytes inside the cavity of the crown ether ring of the CSP has been known to be essential for

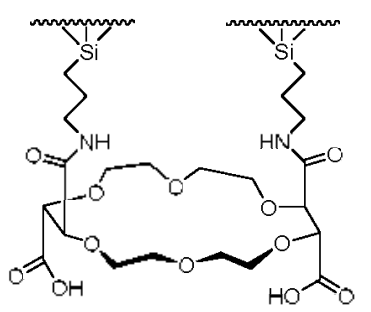

CSP 1

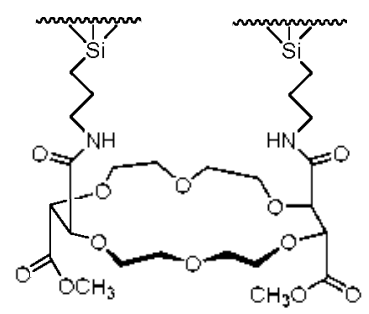

CSP 2

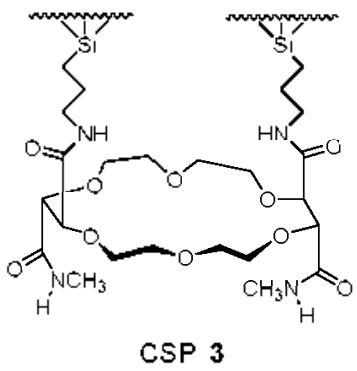

Figure 1. Structures of $\operatorname{CSP} 1, \operatorname{CSP} 2$ and $\operatorname{CSP} 3$ the chiral recognition. ${ }^{2 c}$ In addition. the hydrogen bonding between the carboxylic acid of $(+)-(18-$ crown- 6$)-2,3.11$, 12-tetracarboxylic acid and the carbonyl oxygen of $\alpha$-amino acids has been reported to be essential for the chiral recognition of $\alpha$-amino acids by the NMR study: ${ }^{9}$ Based on the NMR study, the lydrogen bonding between the carboxylic acid of CSP 1 and the carbonyl oxygen of $\alpha$-amino acids has been proposed to be crucial for the chiral recognition of $\alpha$ anino acids. ${ }^{11}$ However, the role of the carboxylic acid groups of the CSP might be different from that of free $(+)-(18-$-crown- 6$)-$ 2.3.11.12-tetracarboxylic acid explored by the NMR study. In this study: we wish to explore whether the two free carboxylic acid groups of CSP 1 are essential or not for the chiral recognition of $\alpha$-amino acids.

In order to explore the role of the two carboxylic acids of CSP 1, we freshly prepared CSP 1 and then divided it into three portions. One portion was used for the column packing. Each of the other two portions was treated with methanol or methylamine in the presence of a coupling agent EEDQ (2-ethoxy-1-ehoxycarbonyl-1.2-dihydroquinoline) to afford CSP 2 and CSP 3 (Figure 1). The two free carboxylic acid groups in CSP 1 are converted to methyl ester groups in CSP 2 or $N$-methylanide groups in CSP 3 . By comparing the chromatographic behaviors of CSP 1, CSP 2 and CSP 3 for the

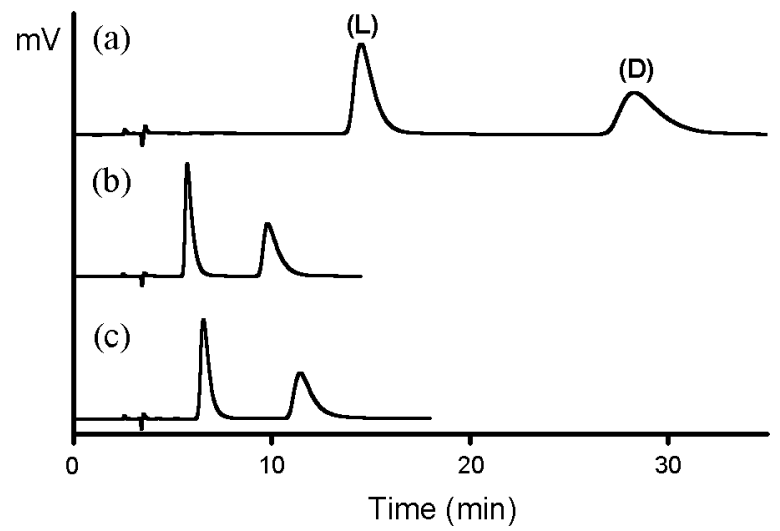

Figure 2. Chromatograms for the resolution of phenylglycine (Pgy) on (a) CSP 1, (b) CSP 2 and (c) CSP 3. For the chromatographic condition, see the footnote to Table 1. 
resolution of $\alpha$-amino acids, we expected that the role of the two carbonylic acid groups of CSP 1 would be elucidated.

The chromatographic results for the resolution of $\alpha$-amino acids on CSP 1, CSP 2 and CSP 3 obtained with the use of a mixed solvent of methanol-water $(80: 20, \mathrm{v} / \mathrm{v})$ containing 10 $\mathrm{mM}$ sulfuric acid as a mobile phase are summarized in Table 1. As an example. the representative chromatograms for the resolution of phenylglycine on CSP 1. CSP 2 and CSP 3 are shown in Figure 2. The elution orders shown in Table 1 and Figure 2 were detenmined by injecting configurationally known samples.

As shown in Table 1. the retention factors $\left(k_{1}\right)$ for the first eluted enantiomers decreased quite much when the carboxylic acid groups of CSP 1 were converted to methyl ester groups (CSP 2) or to $N$-methyl amide groups (CSP 3). The graphical comparison of the retention factors $\left(k_{1}\right)$ for the resolution of $\alpha$ amino acids on CSP 1. CSP 2 and CSP 3 shown in Figure $3 a$ clearly demonstrates the significant difference in the retention factors $\left(k_{1}\right)$.

The carboxylic acid groups of CSP 1 are expected to contribute to the effective hydrogen bonding interaction with analytes. However by converting the carboxylic acid groups of CSP 1 to the methyl ester or N-methyl amide groups, the hydrogen bonding interaction is expected to be diminished quite much and consequently. the retention factors $\left(k_{1}\right)$ on CSP 2 or CSP 3 should decrease compared to those on CSP 1. Retention factors $\left(k_{1}\right)$ on CSP 3 are slightly greater than those on CSP 2. The electron density of the carbonyl oxygen of the $N$-methyl amide group is greater than that of the carbonyl oxygen of the methyl ester group. Consequently, the hydrogen bonding interaction between the carbonyl oxygen of the $N$-methyl amide group of CSP 3 and the analytes might be greater than that between the carbonyl oxygen of the methyl ester group of CSP 2 and the analytes. In addition. the N-H hydrogen of the $N$-methyl amide group of CSP 3 can provide additional hydrogen bonding interaction with the analytes. In this instance, the retention factors ( $k_{1}$ ) on CSP 3 are expected to be greater than those on CSP 2

In contrast to the retention factors $\left(k_{1}\right)$. the selectivity factors ( $\alpha$ ) on CSP 1 are not so much different from those on $\operatorname{CSP} 2$ or CSP 3 as shown in Table 1 and Figure 3b. In some cases, the selectivity factors ( $\alpha$ ) on CSP 2 or CSP 3 are even greater than those on CSP 1 . These very surprising results indicate that the hydrogen bonding between the carboxylic acids of the CSP and the carbonyl oxygen of $\alpha$-anino acids, which has been proposed to be cnucial for the chiral recognition, is not essential for the chiral recognition in the
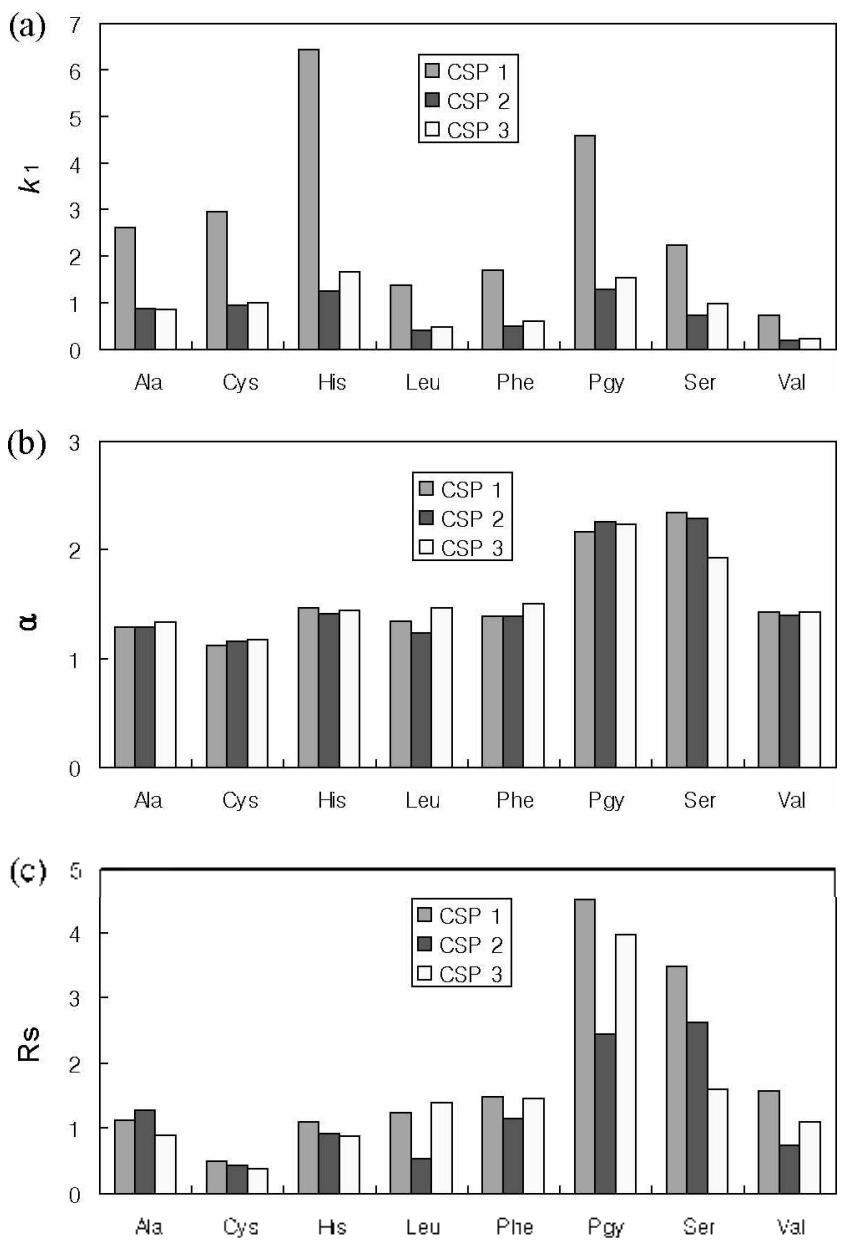

Figure 3. Graphical comparison of (a) the retention factors ( $\left.k_{1}\right)$. (b) the selectivity factors $(\alpha)$ and $(c)$ the resolutions $\left(R_{S}\right)$ for the resolution of $\alpha$-amino acids on CSP $1, \operatorname{CSP} 2$ and CSP 3 .

Table 1. Comparison of the resolution of $\alpha$-annino acids on CSP 1, CSP 2 and CSP $3^{\circ}$

\begin{tabular}{|c|c|c|c|c|c|c|c|c|c|}
\hline & \multicolumn{3}{|c|}{ CSP 1} & \multicolumn{3}{|c|}{ CSP 2} & \multicolumn{3}{|c|}{$\mathrm{CSP} 3$} \\
\hline & $k_{1}$ & $\alpha$ & $\mathrm{R}_{\mathrm{s}}$ & $k_{1}$ & $a$ & $\mathrm{R}_{\mathrm{s}}$ & $k_{1}$ & $\alpha$ & $\mathrm{R}_{s}$ \\
\hline Ala & $2.59(\mathrm{~L})$ & 1.29 & 1.13 & $0.88(\mathrm{~L})$ & 1.29 & 1.28 & $0.86(\mathrm{~L})$ & 1.33 & 0.89 \\
\hline Cys & $2.95(\mathrm{~L})$ & 1.12 & 0.49 & $0.93(\mathrm{~L})$ & 1.16 & 0.42 & $1.01(\mathrm{~L})$ & 1.17 & 0.38 \\
\hline His & $6.42(\mathrm{~L})$ & 1.46 & 1.10 & $1.24(\mathrm{~L})$ & 1.41 & 0.91 & $1.65(\mathrm{~L})$ & 1.44 & 0.87 \\
\hline Lell & $1.38(\mathrm{~L})$ & 1.34 & 1.24 & $0.41(\mathrm{~L})$ & 1.24 & 0.54 & $0.46(\mathrm{~L})$ & 1.46 & 1.39 \\
\hline Pgy & $4.58(\mathrm{~L})$ & 2.16 & 4.50 & $1.28(\mathrm{~L})$ & 2.26 & 2.44 & $1.54(\mathrm{~L})$ & 2.23 & 3.97 \\
\hline Phe & $1.69(\mathrm{~L})$ & 1.38 & 1.49 & $0.50(\mathrm{~L})$ & 1.39 & 1.14 & $0.59(\mathrm{~L})$ & 1.51 & 1.46 \\
\hline SeI & $2.24(\mathrm{D})$ & 2.34 & 3.47 & $0.72(\mathrm{D})$ & 2.29 & 2.63 & $0.96(\mathrm{D})$ & 1.92 & 1.59 \\
\hline Val & $0.71(\mathrm{~L})$ & $1.4 \hat{3}$ & 1.57 & $0.20(\mathrm{~L})$ & 1.40 & 0.73 & $0.22(\mathrm{~L})$ & 1.43 & 1.10 \\
\hline
\end{tabular}

"Mobile phase: $80^{\circ}$ i methanol in water $-10 \mathrm{mM}$ sulfuric acid. Flow rate: $0.5 \mathrm{~mL}$ min. Detection: $210 \mathrm{~nm}$ UV. Temperature: $20^{\circ} \mathrm{C}$. $k_{1}$ : Retention factor of the first eluted enantiomer. The absolute configuration of the first eluted enantiomer is presented in the parenthesis. $\alpha$ : Selectivity factor. $R_{\mathcal{S}}$ : Resolution. Ala: Alanine. Cys: Cystein. His: Histidine, Leu: Leucine. Pgy: Phenylglycine, Phe: Phenylalanine, Ser: Serine, Val: Valine. 
resolution of $\alpha$-amino acids on CSP 1 . In addition. the elution orders were identical on the three CSPS as shown in Table 1. indicating that the chiral recognition mechanisms are quite similar or identical on the three CSPs. Consequently. we expect that the two carboxylic acids groups of CSP 1 are not working as enantioselective hydrogen bonding sites even though they are working as non-enantioselective hydrogen bonding sites. Instead, each of the free carboxylic acid groups of CSP 1, the methyl ester groups of CSP 2 or the 1 -methyl amide groups of CSP 3 do play a quite similar role such as chiral barrier sites for the chiral recognition. At this point it should be noted that the hydrogen bonding interactions between the carbonyl oxygen of the methyl ester group of CSP 2 or the 1 -methyl amide group of CSP 3 and the analytes are also non-enanatioselective and consequently they are not responsible for the chiral recognition, but responsible only for the retention of analytes.

Resolutions ( $\mathrm{R}_{S}$ ) on CSP 1 are generally greater than those on CSP 2 or CSP 3 except for alanine and leucine as shown in Table 1 and Figure $3 \mathrm{c}$. The relatively short retention times on CSP 2 and CSP 3 were expected to make the resolution ( $\left.R_{S}\right)$ on these CSPs worse compared to those on CSP 1

In conclusion, in this study, we demonstrated that the free carboxylic acid groups of CSP 1 are not essential for the chiral recognition. Instead, the carboxylic acid groups of CSP 1 might play a role as chiral barrier sites for the chiral recognition as the methyl ester groups of CSP 2 or the 1 -methyl amide groups of CSP 3 might do

\section{Experimental}

General. Chromatography was performed with an HPLC system consisting of a Waters model 510 HPLC Pump. a Rheody ne model $7725 \mathrm{i}$ injector with a $20 \mu \mathrm{L}$ sample loop. a Waters 2487 Absorbance Detector and a YoungLin Autochro Data Module (Software: YoungLin Autochro-WIN 2.0 plus). The temperature of the chiral column was maintained at $20^{\circ} \mathrm{C}$ by using a Julabo F30 Ultratemp 2000 cooling circulator.

Racemic and optically active $\alpha$-amino acids used in this study were available from Aldrich. Each of racemic and optically active samples was dissolved in methanol (usulally $1.0 \mathrm{mg} / \mathrm{mL}$ ) and then used for the resolution on the CSPs. The usual injection volume was $3.0 \mu \mathrm{L}$

Preparation of CSPs and column packing. CSP 1 was prepared by bonding $(+)-(18-$-crown- 6$)-2.3 .11$.12-tetracarboxylic acid ( $1.0 \mathrm{~g} .2 .28 \mathrm{mmol}$ ) to aminopropylsilica gel (7.9 g. Kromasil $5 \mu \mathrm{m}$. 100 A) via the same procedure as that reported previously except with the use of 2.6-lutidine instead of triethylamine. ${ }^{3 a}$ The elemental analysis of aminopropylsilica gel (C. $5.80 \%: H, 1.47 \%:$ N. $2.00 \%$ ) and CSP 1 (C. $6.55 \%: \mathrm{H}, 1.74 \%: \mathrm{N}, 1.96 \%$ ) showed that the loading of $(+)-$ (18-crown-6)-2.3,11.12-tetracarboxylic acid per gram of aminopropylsilica gel was $0.039 \mathrm{mmol}$ based on carbon.

CSP 1 thus prepared was divided into three portions. One portion was reserved for column packing. Each $(2.5 \mathrm{~g})$ of the other two portions of CSP 1 was suspended in toluene $(60 \mathrm{~mL})$ in $150 \mathrm{~mL}$ two-neck round bottom flask equipped with a
Dean-Stark trap, a condenser and a magnetic stirrer. The heterogeneous mixture was heated to reflux to remove water azeotropically. After the complete azeotropic removal of water. toluene was removed by rotary evaporation. To the residue were added anhydrous methanol $(30 \mathrm{~mL})$ and EEDQ $\left(0.35 \mathrm{~g}\right.$. $1.42 \mathrm{mmol}$ ). The whole nuixture was stirred at $0^{\circ} \mathrm{C}$ for two days. The modified silica gel (CSP 2) was washed successively with methanol acetone, ethyl acetate. methylene clloride. hexane and ether and then dried under high vacuum. Based on the elemental analy'sis of CSP 1 and CSP $2(\mathrm{C}, 6.64 \%: \mathrm{H} .1 .82 \%$. N. $1.94 \%$ ). methanol used for the formation of methyl ester groups of CSP 2 was calculated to be $0.083 \mathrm{mmol}$ per gram of CSP 1 based on the carbon.

CSP 3 was prepared by treating CSP 1 with methylamine in the presence of EEDQ via the same procedure for the preparation of CSP 2. Based on the elemental analysis of CSP 1 and CSP $3(\mathrm{C} .6 .64 \%:$ H. 1.84\%: N. 2.06\%), methylamine used for the formation of $N$-methyl amide groups of CSP 3 was calculated to be $0.075 \mathrm{mmol}$ per gram of CSP 1 based on the carbon. Each of three CSPs thus prepared (CSP 1, CSP 2 and CSP 3) was slurried in methanol and packed into $150 \times 4.6 \mathrm{~nm}$ I.D. stainless steel HPLC column by using a conventional slurry packing method with an Alltech slurry packer.

Aclonowledgments. This work was supported by the grant (No. RTI04-02-01) from the Regional Teclunology Innovation Program of the Ministry of Commerce. Industry and Energy. Korea and by the grant (No. R-15-2006-022-03001-0) for the National Core Research Center Program from MEST/KOSEF.

\section{Refeiences}

1. (a) Hyur, M. H. J. Sep. Sci. 2003, 26, 242. (b) Hyum, M. H. Bull. Kor: Chem. Soc. 2005, 26, 1153 (c) Choi. H. T.: Hyun. M. H. J. Liq. Chromatogr: Rel. Technol. 2007,30,853. (d) Hyun, M. H. In Chiral Separation Techinques: A Practical Approach. Third Contpletely Revised and Lpdated Edition; Subramanian, G., Ed.; Wiley-VCH: Weinheim, 2007; Chap. 8

2. (a) Machida, Y.: Nishi, H.: Nakamura, K.: Nakai, H.: Sato, T. J. Chomatogr. A 1998, 805. 82. (b) Hyun, M. H. Tin. T. S.; Lee, W. Bull Kor: Chem. Soc. 1998, 19,819. (c) Hyun, M. H.; Hyun, M. H. J. Sep. Sci. $2006,29,750$.

3. (a) Hyun, M. H.; Jin, T. S.; Lee, W. J. Chromatogr . A 1998, 822, 155. (b) Hyun, M. H.; Cho, Y. J.; Tin, T. S. J. Sep. Sci. 2002, 25, 648. (c) Jin, J. Y.: Baek, C.-S.: Lee, W. Bull Kor. Chem. Soc. 2007, 28, 1070. (d) Lee, S. J.: Cho, H. S.; Choi, H. J.: Hyun, M. H. J. Chromatogr. A 2008, 1188, 318.

4. Hyun, M. H.; Tan, T; Cho, Y. J. J. Liq. Chromatogr. Ret. Techol. 2004, 27, 1671

5. Hylun, M. H.; Min. H. J.: Cho, Y. J. Bull. Kor Chem. Soc. 2003. 24.911

6. Hyun, M. H.; Jin, J. S.: Koo, H. T.; Lee, W. J. Chromatogr At $1999,837,75$.

7. Conrad, U.; Chankwetadze, B.; Scriba, G. K. E. J. Sep. Sci. 2005, 28,2275

8. (a) Hyun, M. H.: Han, S. C. Tin, I. S.: Lee, W. Chromatographia 2000, 52, 473, (b) Hyun, M. H.; Han, S. C.; Cho, Y. J.: Jin, I. S.; Lee, W. Bionted. Chromatogr. 2002, 16, 356.

9. Bang, E.; Jung, J.W.; Lee, W.; Lee, D. W; Lee, W. J. Chem. Soc, Perkin Trans 22001,1685

10. Lee, W.: Jin, I. Y.: Baek, C.-S. M ficrochem. J. 2005, 80,213 